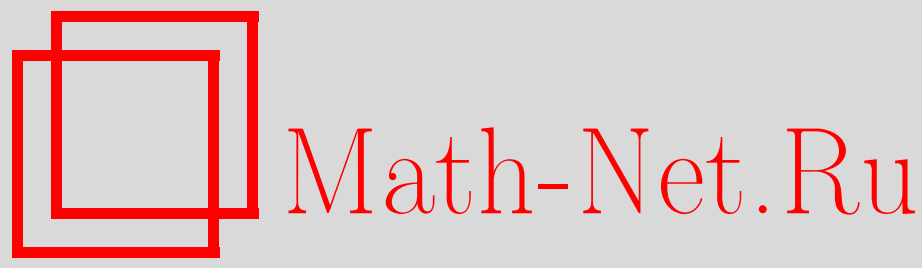

В. А. Малышев, С. А. Музычка, Динамический фазовый переход в простейшей модели цепочки молекул, ТМФ, 2014, том 179, номер 1, 123-133

DOI: https://doi.org/10.4213/tmf8608

Использование Общероссийского математического портала Math-Net.Ru подразумевает, что вы прочитали и согласны с пользовательским соглашением http://www.mathnet.ru/rus/agreement

Параметры загрузки:

IP : 3.80 .181 .102

26 апреля 2023 г., 12:43:14

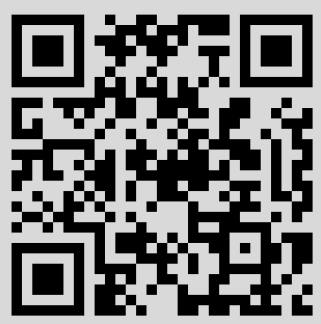




\section{ДИНАМИЧЕСКИЙ ФАЗОВЫЙ ПЕРЕХОД В ПРОСТЕЙШЕЙ МОДЕЛИ ЦЕПОЧКИ МОЛЕКУЛ}

Рассматривается динамика простейшей цепочки из большого числа $N$ частиц. В двойном масштабном пределе найдено разбиение пространства параметров на две области, в одной из которых точная верхняя грань по интервалу времени $(0, \infty)$ относительного удлинения цепочки при $N \rightarrow \infty$ стремится к единице, а в другой - к бесконечности.

Ключевые слова: статистическая физика, двойной масштабный предел, динамический фазовый переход, закон Гука, молекулярная цепочка.

DOI: $10.4231 / \operatorname{tmf} 8608$

\section{1. ВВЕДЕНИЕ}

Для математических моделей равновесной статистической физики необходима устойчивость, т.е. конечность статистической суммы в конечном объеме. Условие устойчивости дает хорошее приближение для многих явлений в газах, жидкостях и даже твердых телах. Однако, например, исследование моделей расширения или разрушения твердых тел упирается в то, что объем не фиксирован, и часто необходимо рассматривать конечное число частиц в бесконечном объеме. При реалистических взаимодействиях (когда взаимодействие исчезает на бесконечности) такая система не является устойчивой с точки зрения распределения Гиббса. При этом говорят, что система является метастабильной [1]. В этом случае для конечного числа частиц необходимо доказывать, что система не выходит из определенной области фазового пространства (не распадается на части). Так как эта область зависит от всех параметров модели, при большом числе частиц удобно применять прием, который в физике называют иногда двойным масштабным пределом (double scaling limit), или двойным скейлингом, например, в случае, когда все параметры зависят от числа частиц. Тогда можно устремлять число частиц к бесконечности и получать точные асимптотические оценки в таком термодинамическом пределе.

Мы рассматриваем одномерную систему из $N$ частиц (молекул) одинаковой массы $m$, причем в начальный момент $t=0$ координаты частиц таковы:

$$
0=z_{0}(0)<z_{1}(0)=a<z_{2}(0)=2 a<\cdots<z_{N-1}(0)=(N-1) a
$$

* Московский государственный университет им. М.В. Ломоносова, Москва, Россия. E-mail: malyshev2@yahoo.com, stepan_muzychka@mail.ru 
для некоторого $a>0$. Динамика этой системы определяется гамильтонианом

$$
H=\sum_{k=1}^{N-1} \frac{p_{k}^{2}}{2 m}+\sum_{k=1}^{N-1} V\left(z_{k}-z_{k-1}\right)-f z_{N-1} .
$$

Предполагается, что частица $z_{0}$ постоянно закреплена в нуле, а на частицу $z_{N-1}$ действует постоянная внешняя сила $f>0$. Относительно функции $V(z)$ обычно считается, что $V(z) \rightarrow \infty$ при $z \rightarrow 0$ и $V(z) \rightarrow 0$ при $z \rightarrow \infty$, кроме того, функция $V(z)$ выпукла на интервале $(0, b)$, вогнута на интервале $(b, \infty)$ и имеет единственный минимум $V(a)<0$ в точке $a>0$, причем $a<b<\infty$. Мера Гиббса с таким гамильтонианом, конечно, не существует и возможны два пути решения этой проблемы.

1. Изменить $V(z)$ при больших $z$ так, чтобы $V(z) \rightarrow \infty$ при $z \rightarrow \infty$. Тогда можно применять гиббсовскую идеологию в условиях равновесия для исследования температурного и упругого расширения (см. книгу [2], где можно найти много прикладных задач на эту тему, и, в частности, простым вычислением показан линейный рост по $T$ дисперсии температурного расширения у гармонической цепочки). Однако, как показано ранее в работе [3], среднее расширение может быть линейным по $T$ (для низких температур $T$ ) только в негармоническом случае.

2. Найти окрестность $O(a)$ точки минимума такую, что при начальных данных (1) траектория никогда не выходит из $O(a)$.

В настоящей работе мы пойдем по второму пути, предполагая дополнительно, что в некоторой окрестности точки $a$ потенциал имеет квадратичный вид:

$$
V(z)=\frac{\kappa}{2}(z-a)^{2} .
$$

Теперь сформулируем более точные утверждения. Нетрудно вычислить растяжение такой цепочки в статической ситуации (при нулевой температуре), а именно найти единственную неподвижную точку динамики (см. ниже). Однако логично исследовать также динамику системы частиц во времени и получить хорошие оценки для функционала

$$
A=A(N, l, f, \kappa, m)=\sup _{t \in(0, \infty)} \max _{0 \leqslant k, k+l \leqslant N-1}\left|z_{k+l}(t)-z_{k}(t)\right|
$$

для больших $N$ и различных $l>0$. Несмотря на очевидную простоту модели, основной результат настоящей статьи - оценка максимума (по всему бесконечному интервалу времени) отклонений от исходной “кристаллической” структуры - является нетривиальным и получается с использованием теоретико-числовых оценок. Дело в том, что, хотя в нашей модели есть очевидная неподвижная точка, но модель гамильтонова, поэтому никакой сходимости к этой точке нет. Отсюда возникает задача оценить, насколько далеко траектория отходит от этой неподвижной точки. Мы начинаем с фиксированного числа $N$ частиц и находим окрестность, из которой система никогда не выходит. Затем, устремляя $N \rightarrow \infty$ и делая скейлинг параметров, мы обнаруживаем, что имеется фазовый переход, разделяющий область, где кристаллическая структура мало меняется на протяжении всего бесконечного времени, и область, где точная верхняя грань растет с ростом $N$.

Заметим, что есть много работ, посвященных другим задачам для одномерных моделей. Одни из наиболее известных - модели Ферми-Паста-Улама [4] и модель 
Френкеля-Конторовой [5]. Следует отметить также работы [6]-[9], берущие начало от монографии [10], где многомерные статические модели рассматривались с целью вывода уравнений линейной упругости из микромоделей.

\section{2. ОСНОВНОЙ РЕЗУЛЬТАТ}

Вводя отклонения $x_{k}(t)=z_{k}(t)-k a, k=0, \ldots, N-1$, имеем гамильтонову систему линейных уравнений

$$
\begin{aligned}
\ddot{x}_{0}(t) & =0, \\
\ddot{x}_{k}(t) & =\omega_{0}^{2}\left(x_{k-1}-2 x_{k}+x_{k+1}\right), \quad k=1, \ldots, N-2, \\
\ddot{x}_{N-1}(t) & =\omega_{0}^{2}\left(-x_{N-2}+x_{N-1}\right)+f_{0}
\end{aligned}
$$

с начальными данными $(1)$ и $v_{k}(0)=\dot{x}_{k}(0)=0, k=1, \ldots, N-1$. Мы ввели обозначение $\omega_{0}^{2}=\kappa / m$ для собственной частоты осцилляторов и положили $f_{0}=f / m$.

Определим вспомогательную функцию

$$
F_{N}(x)=x \ln \frac{N}{x}, \quad x>0,
$$

и введем наш основной параметр $\sigma=f / \kappa=f_{0} / \omega_{0}^{2}$. Условимся, что константы, обозначаемые далее как $c, c_{i}$, const, не зависят от $N, l, f_{0}, \omega_{0}$ и $a$. Основная оценка такова.

ТЕОрема 1. Пусть $\varepsilon \in(0,1)$ - произволъное число, тогда для всех $k, l \in \mathbb{N}$, удовлетворяющих условию

$$
0 \leqslant k<k+l \leqslant(1-\varepsilon) N
$$

имеют место неравенства

$$
\begin{aligned}
& \sigma\left(l+c_{1} F_{N}(l)\right) \leqslant \sup _{t \geqslant 0}\left(x_{k+l}(t)-x_{k}(t)\right) \leqslant \sigma\left(l+c_{2} F_{N}(l)\right), \\
& \sigma\left(l-c_{3} F_{N}(l)\right) \leqslant \inf _{t \geqslant 0}\left(x_{k+l}(t)-x_{k}(t)\right) \leqslant \sigma\left(l-c_{4} F_{N}(l)\right)
\end{aligned}
$$

для некоторых положительных $c_{1}, c_{2}, c_{3}, c_{4}$, причем $c_{1}$ и $c_{3}$ могут зависеть от $\varepsilon$.

Далее мы используем процедуру, которая в физике иногда называется двойной скейлинг. А именно, мы положим $a=1 / N$ и будем рассматривать различные скейлинги $l=l(N)$ и $\sigma=\sigma(N)$.

Далее мы используем следующее обозначение: для положительных функций будем писать $f(x) \simeq g(x)$, если существуют такие $c_{1}, c_{2}>0$, что на всей области определения $c_{1} g(x) \leqslant f(x) \leqslant c_{2} g(x)$.

СлЕДСТвИЕ 1. В условиях теоремъ 1

$$
\left|x_{k+l}(t)-x_{k}(t)\right| \simeq \sigma(N) l \ln \frac{N}{l} .
$$


В качестве характеристики фазового перехода рассмотрим максимальное относительное удлинение (для $l=1$ )

$$
\frac{A}{a}=N A
$$

под действием силы $f$. При этом

$$
\begin{array}{lllll}
\frac{A}{a} \rightarrow 1, & \text { если } & \sigma(N) N \ln N \rightarrow 0 & \text { при } & N \rightarrow \infty, \\
\frac{A}{a} \rightarrow \infty, & \text { если } & \sigma(N) N \ln N \rightarrow \infty & \text { при } & N \rightarrow \infty .
\end{array}
$$

Сравнение с фазовым переходом в состоянии равновесия. В случае квадратичного гамильтониана (3) неподвижная точка всегда существует и единственна, причем для всех $k$ будет выполнено равенство $z_{k}-z_{k-1}=h=a+\sigma$. Поэтому здесь $h / a \rightarrow \infty$, если $\sigma N \rightarrow \infty$, и $h / a \rightarrow 1$, если $\sigma N \rightarrow 0$. Таким образом, различие между динамическим и статическим фазовыми переходами определяется логарифмическим множителем.

Аналогичный факт имеет место для более общих взаимодействий. Обычно взаимодействие берется в виде

$$
V(r)=-\frac{c_{n}}{r^{n}}+\frac{c_{m}}{r^{m}}
$$

Заметим, что для произвольных $0<n<m$ и $c_{n}>0, c_{m}>0$ функция $V(r)$ удовлетворяет сформулированным выше свойствам и имеет место следующая картина. Если

$$
\max _{a<h \leqslant b} \frac{d V(h)}{d h} \geqslant f
$$

то гамильтонова динамика (2) имеет неподвижную точку, для которой при всех $k$ выполнены неравенства $b \geqslant z_{k}-z_{k-1}=h>a>0$, а значение $h$ определяется из уравнения

$$
\frac{d V(h)}{d h}=f
$$

Если же

$$
\max _{a<h \leqslant b} \frac{d V(h)}{d h}<f,
$$

то неподвижной точки не существует, и под действием силы $f$ цепочка распадается.

Следующее утверждение касается статического фазового перехода для взаимодействий (10).

Лемма 1. Если $a=1 / N$, то неподвижная точка существует тогда и только тогда, когда

$$
\sigma=\frac{f}{\kappa} \leqslant \frac{1}{N} C
$$

где

$$
\kappa=V^{\prime \prime}(a), \quad C=C(n, m)=\frac{1}{m-n}\left(\left(\frac{m+1}{n+1}\right)^{-\frac{n+1}{m-n}}-\left(\frac{m+1}{n+1}\right)^{-\frac{m+1}{m-n}}\right) .
$$

ЗАмЕчАниЕ 1. В случае, когда температура равна нулю, в предположении, что $V(x) \rightarrow \infty$ при $x \rightarrow \infty$, распределение Гиббса существует. При этом существование температурного и упругого расширения в равновесном случае зависит от вида функции $V$ в окрестности минимума [3]. 


\section{3. ДОКАЗАТЕЛЬСТВА}

3.1. Вспомогательные утверждения. Докажем несколько вспомогательных утверждений, необходимых при доказательстве основной теоремы.

Лемма 2. Система уравнений (5) имеет решение

$$
x_{n}(t)=\sigma\left(n-\frac{1}{2 N-1} \sum_{m=1}^{2 N-2} \gamma_{m, N, n} \cos \omega_{m} t\right)
$$

¿əe

$$
\gamma_{m, N, n}=\sin ^{-2} \frac{\pi m}{4 N-2} \sin \frac{\pi m}{2} \cos \frac{\pi m}{4 N-2} \sin \frac{\pi n m}{2 N-1}, \quad \omega_{m}=2 \omega_{0} \sin \frac{\pi m}{4 N-2} .
$$

ДокАЗАтельство. Рассмотрим следующую вспомогательную систему из $4 N-2$ уравнений на окружности (т. е. сложение и вычитание индексов у функций ведется по модулю $4 N-2)$ :

$$
\begin{array}{r}
\ddot{y}_{n}=\omega_{0}^{2}\left(y_{n-1}-2 y_{n}+y_{n+1}\right)+f_{0}\left(\delta_{n, N-1}+\delta_{n, N}-\delta_{n, 3 N-2}-\right. \\
\left.\delta_{n, 3 N-1}\right), \\
n=0, \ldots, 4 N-3,
\end{array}
$$

с нулевыми начальными условиями. Мы утверждаем, что для всех $t$

$$
x_{n}(t) \equiv y_{n}(t), \quad n=0, \ldots, N-1 .
$$

Действительно, во-первых, из симметричности уравнений следует, что

$$
y_{n}=y_{2 N-1-n}=-y_{-n}=-y_{2 N-1+n}, \quad n=0, \ldots, N-1 .
$$

Таким образом, $\ddot{y}_{0}=\omega_{0}^{2}\left(y_{-1}-2 y_{0}+y_{1}\right)=-2 \omega_{0}^{2} y_{0}$, откуда с учетом нулевых начальных условий следует, что $y_{0}(t)=x_{0}(t) \equiv 0$. Далее,

$$
\ddot{y}_{n}=\omega_{0}^{2}\left(y_{n-1}-2 y_{n}+y_{n+1}\right), \quad n=1, \ldots, N-2 .
$$

И наконец, при $n=N-1$

$$
\ddot{y}_{N-1}=\omega_{0}^{2}\left(y_{N-2}-2 y_{N-1}+y_{N}\right)+f_{0}=\omega_{0}^{2}\left(y_{N-2}-y_{N-1}\right)+f_{0} .
$$

Таким образом, системы дифференциальных уравнений для $x_{n}$ и $y_{n}$ полностью совпадают, откуда следует требуемое утверждение.

В свою очередь, система уравнений (14) решается при помощи преобразования Фурье:

$$
x_{n}=y_{n}=\frac{1}{4 N-2} \sum_{m=0}^{4 N-3} \alpha_{m} e^{-\frac{2 \pi i n m}{4 N-2}}
$$

где

$$
\alpha_{m}=\sum_{n=0}^{4 N-3} y_{n} e^{\frac{2 \pi i n m}{4 N-2}}
$$


является решением соответствующей системы дифференциальных уравнений с нулевыми начальными условиями:

$$
\begin{aligned}
\ddot{\alpha}_{m}+\omega_{m}^{2} \alpha_{m} & =f_{0}\left(e^{\frac{2 \pi i m(N-1)}{4 N-2}}+e^{\frac{2 \pi i m N}{4 N-2}}-e^{\frac{2 \pi i m(-N+1)}{4 N-2}}-e^{-\frac{2 \pi i m N}{4 N-2}}\right)= \\
& =f_{0}\left(e^{\frac{\pi i m}{2}}-e^{-\frac{\pi i m}{2}}\right)\left(e^{\frac{\pi i m}{4 N-2}}+e^{-\frac{\pi i m}{4 N-2}}\right)=4 i f_{0} \cos \frac{\pi m}{4 N-2} \sin \frac{\pi m}{2} .
\end{aligned}
$$

Отсюда следует, что

$$
\begin{aligned}
\alpha_{m}(t) & =\frac{4 i f_{0}}{\omega_{m}^{2}} \cos \frac{\pi m}{4 N-2} \sin \frac{\pi m}{2}\left(1-\cos \omega_{m} t\right)= \\
& =\frac{i f_{0}}{\omega_{0}^{2}} \sin ^{-2} \frac{\pi m}{4 N-2} \cos \frac{\pi m}{4 N-2} \sin \frac{\pi m}{2}\left(1-\cos \omega_{m} t\right) .
\end{aligned}
$$

Поскольку $y_{n}=-y_{-n}$, мы имеем $\alpha_{m}=-\alpha_{-m}$, поэтому

$$
\begin{aligned}
x_{n}=y_{n} & =\frac{1}{4 N-2} \sum_{m=0}^{2 N-2} \alpha_{m}\left(e^{\frac{-2 \pi i n m}{4 N-2}}-e^{\frac{2 \pi i n m}{4 N-2}}\right)= \\
& =-\frac{2 i}{4 N-2} \sum_{m=1}^{2 N-2} \alpha_{m} \sin \frac{\pi n m}{2 N-1} .
\end{aligned}
$$

В итоге получаем формулу

$$
y_{n}=\frac{\sigma}{2 N-1} \sum_{m=1}^{2 N-2} \gamma_{m, N, n}\left(1-\cos \omega_{m} t\right)
$$

Чтобы завершить доказательство, остается проверить, что

$$
\frac{1}{2 N-1} \sum_{m=1}^{2 N-2} \gamma_{m, N, n}=n
$$

Рассмотрим ту же систему (14), но с другими начальными условиями

$$
y_{n}=y_{2 N-1-n}=n \sigma, \quad n=-N+1, \ldots, N-1 \text {. }
$$

Нетрудно проверить, что в данном случае система находится в состоянии равновесия: $y_{n}(t) \equiv y_{n}(0)$. Как следствие, $\alpha_{m}(t)$ также не меняется во времени. Из уравнений (15) следует, что такое возможно только при

$$
\alpha_{m}(0)=\frac{4 i f_{0}}{\omega_{m}^{2}} \cos \frac{\pi m}{4 N-2} \sin \frac{\pi m}{2}=i \sigma \sin ^{-2} \frac{\pi m}{4 N-2} \cos \frac{\pi m}{4 N-2} \sin \frac{\pi m}{2},
$$

и потому из (16) вытекает, что

$$
n \sigma=x_{n}(0)=-\frac{2 i}{4 N-2} \sum_{m=1}^{2 N-2} \alpha_{m}(0) \sin \frac{\pi n m}{2 N-1}=\frac{\sigma}{2 N-1} \sum_{m=1}^{2 N-2} \gamma_{m, N, n} .
$$

Отсюда следует равенство (17). Лемма доказана. 
Прямая подстановка формулы (12) показывает, что имеет место следующее тождество:

$$
I_{N, k, l}(t):=\frac{1}{\sigma}\left(x_{k+l}(t)-x_{k}(t)-\sigma l\right)=-\frac{2}{2 N-1} \sum_{m=1}^{2 N-2} a_{m} b_{m} \cos \omega_{m} t
$$

где

$$
a_{m}=\sin ^{-2} \frac{\pi m}{4 N-2} \sin \frac{\pi m}{2} \cos \frac{\pi m}{4 N-2} \sin \frac{\pi m l}{4 N-2}, \quad b_{m}=\cos \frac{\pi m(k+l / 2)}{2 N-1} .
$$

Проверим несколько вспомогательных фактов относительно $a_{m}$ и $b_{m}$, которые нам понадобятся впоследствии.

ЛЕмма 3. Справедливы следующие утверждения:

1) $\left|b_{m}\right| \leqslant 1$ для всех $m \in \mathbb{Z}$,

2) $a_{m}=0$ для всех четных $m$,

3) $\left|a_{m}\right| \leqslant$ const $\cdot N^{2} / m^{2}$ для всех $1 \leqslant m \leqslant 2 N$,

4) $\left|a_{m}\right| \simeq N l / m$ для всех нечетных $m, 1 \leqslant m \leqslant N / l$,

5) $\left|b_{m}\right| / m+\left|b_{m+2}\right| /(m+2) \geqslant \mathrm{const} / m$ для всех $m>0$.

ДоказАтельство. Первое и второе утверждения очевидны. Третье вытекает из неравенства

$$
\left|a_{m}\right| \leqslant \sin ^{-2} \frac{\pi m}{4 N-2} \leqslant \text { const } \cdot \frac{N^{2}}{m^{2}},
$$

а четвертое есть следствие того, что при $1 \leqslant m \leqslant N / l$

$$
\sin \frac{\pi m}{4 N-2} \simeq \frac{m}{N}, \quad \cos \frac{\pi m}{4 N-2} \simeq 1, \quad \sin \frac{\pi m l}{4 N-2} \simeq \frac{m l}{N} .
$$

Для проверки пятого неравенства покажем, что существует такая постоянная $c>0$, что для любого $m \in \mathbb{Z}$ по крайней мере одно из двух чисел $\left|b_{m}\right|$ или $\left|b_{m+2}\right|$ не меньше $c$. Из условия (7) вытекает, что

$$
2 k+l \leqslant 2(k+l) \leqslant 2(1-\varepsilon) N,
$$

отсюда

$$
0 \leqslant \frac{\pi(2 k+l)}{2 N-1} \leqslant \pi(1-\varepsilon)
$$

а потому функция

$$
g(x)=\min \left(|\cos x|,\left|\cos \left(x+\frac{\pi(2 k+l)}{2 N-1}\right)\right|\right)
$$

не обращается в ноль при всех $x \in \mathbb{R}$. Таким образом, с учетом периодичности этой функции мы имеем $\inf _{x} g(x)>0$. Но

$$
\min \left(\left|b_{m}\right|,\left|b_{m+2}\right|\right)=g\left(\frac{\pi m(k+l / 2)}{2 N-1}\right) \geqslant \inf _{x} g(x)>0,
$$

откуда следует требуемое утверждение. В результате для всех $m>0$

$$
\frac{\left|b_{m}\right|}{m}+\frac{\left|b_{m+2}\right|}{m+2} \geqslant \frac{\inf _{x} g(x)}{m+2} \geqslant \frac{\text { const }}{m} .
$$


Лемма 4. Существует такая константа с >0, что для всех $N \in \mathbb{N}$ при

$$
M=M(N, c)=\left\lfloor\frac{c N}{\ln \ln N}\right\rfloor
$$

числа

$$
\frac{\omega_{1}}{2 \omega_{0}}, \frac{\omega_{2}}{2 \omega_{0}}, \ldots, \frac{\omega_{M}}{2 \omega_{0}}
$$

рационально независимь, т.е. не существует таких $a_{0}, a_{1}, \ldots, a_{M} \in \mathbb{Z}$, что

$$
\sum_{m=1}^{M} a_{m} \frac{\omega_{m}}{2 \omega_{0}}=a_{0}
$$

ДокАзАТЕЛЬство. При доказательстве нам потребуются следующие факты из теории чисел:

1) алгебраическая степень числа $e^{2 \pi i / n}, n \in \mathbb{N}$, равна $\varphi(n)$, где $\varphi(\cdot)-$ функция Эйлера [11];

2) имеет место следующая асимптотика [12]:

$$
\liminf _{n \rightarrow \infty} \frac{\varphi(n) \ln \ln n}{n}=e^{-\gamma},
$$

где $\gamma$ - постоянная Эйлера-Маскерони.

Перейдем к доказательству утверждения леммы. Предположим противное. Положив $z=e^{\pi i /(4 N-2)}$, перепишем условие $(20)$ рациональной зависимости в виде

$$
\frac{1}{2 i} \sum_{m=1}^{M} a_{m}\left(z^{m}-z^{-m}\right)=a_{0}
$$

следовательно,

$$
\left(\sum_{m=1}^{M} a_{m}\left(z^{m}-z^{-m}\right)\right)^{2}+4 a_{0}^{2}=0
$$

отсюда

$$
\left(\sum_{m=1}^{M} a_{m}\left(z^{M+m}-z^{M-m}\right)\right)^{2}+4 a_{0}^{2} z^{2 M}=0 .
$$

Поскольку в левой части тождества стоит многочлен степени не больше $4 M$, алгебраическая степень числа $z$ не превосходит $4 c N / \ln \ln N$. В то же время из приведенных выше двух утверждений следует, что существует такое число $c^{\prime}>0$, что алгебраическая степень $z$ не меньше $c^{\prime} N / \ln \ln N$. Таким образом, в силу произвольности $c$ получаем противоречие.

СлЕДСТВиЕ 2. В условиях леммы 4 для любъх $a_{1}, \ldots, a_{M} \in \mathbb{R}$

$$
\sup _{t \geqslant 0} \sum_{m=1}^{M} a_{m} \cos \omega_{m} t=-\inf _{t \geqslant 0} \sum_{m=1}^{M} a_{m} \cos \omega_{m} t=\sum_{m=1}^{M}\left|a_{m}\right| .
$$

ДокАЗАТЕЛЬСтво. Из леммы 4 следует, что траектория $\left(\omega_{1} t, \omega_{2} t, \ldots, \omega_{M} t\right)$ всюду плотна на соответствующем $M$-мерном торе $T$, откуда следует требуемое утверждение. 
3.2. Доказательство теоремы 1. Докажем неравенства (8), неравенства (9) доказываются аналогично.

Оценка снизу. Разобьем сумму в (18) на две части,

$$
I_{N, k, l}(t)=I_{N, k, l}^{1}(t)+I_{N, k, l}^{2}(t):=\sum_{m \leqslant M(N)}(\cdot)+\sum_{m>M(N)}(\cdot),
$$

и оценим каждую из них по отдельности.

Для первой суммы из следствия 2 вытекает, что (ниже [·] означает целую часть, $\wedge$ означает минимум, $\vee$ означает максимум)

$$
\begin{aligned}
\sup _{t \geqslant 0} I_{N, k, l}^{1}(t) & =\frac{2}{2 N-1} \sum_{\substack{m=1, \\
\text { нечетное } \\
M(N) \wedge[N / l]}}^{M(N)}\left|a_{m} b_{m}\right| \geqslant \frac{2}{2 N-1} \sum_{\substack{m=1, \\
\text { нечетное }}}^{M(N) \wedge[N / l]}\left|a_{m} b_{m}\right| \geqslant \\
& \left.\geqslant \text { const } \cdot l \cdot \sum_{\substack{m=1, m \text { нечетное } \\
[(M(N) \wedge[N / l]) / 4]}}^{\left[\left(b_{m} \mid\right.\right.} \frac{\left|b_{4 m+1}\right|}{4 m+1}+\frac{\left|b_{4 m+3}\right|}{4 m+3}\right) \geqslant \\
& =\text { const } \cdot l \cdot \sum_{m=1}^{[(M(N) \wedge[N / l]) / 4]} \frac{1}{m} \geqslant \operatorname{const} \cdot l \cdot \ln \frac{M(N) \wedge[N / l]}{4} \geqslant \\
& \geqslant \text { const } \cdot l \cdot \sum_{m=1} \text { const } \cdot l \cdot \ln \left(\operatorname{const} \cdot \frac{N}{l \vee \ln \ln N}\right) \geqslant \operatorname{const} \cdot F_{N}(l) .
\end{aligned}
$$

Здесь в первом неравенстве мы заменили сумму с одним числом слагаемых на сумму с меньшим числом слагаемых. Во втором неравенстве мы воспользовались утверждением 4 леммы 3, а в третьем применили утверждение 5 леммы 3. В четвертом неравенстве мы воспользовались тем, что

$$
\sum_{m=1}^{n} \frac{1}{m} \simeq \ln n, \quad n \in \mathbb{Z}
$$

Пятое неравенство следует из определения (19) функции $M(N)$, а шестое неравенство получается из рассмотрения двух отдельных случаев: при $l \geqslant \ln \ln N$ левая часть неравенства в точности совпадает с правой, а при $l<\ln \ln N$

$$
l \ln \left(\text { const } \cdot \frac{N}{\ln \ln N}\right)=l(\text { const }+\ln N-\ln \ln \ln N) \simeq l \ln N \geqslant F_{N}(l) .
$$

Перейдем к оценке второй суммы. Имеем

$$
\begin{aligned}
\sup _{t \geqslant 0}\left|I_{N}^{2}(k, l)\right| & \leqslant \text { const } \cdot N \cdot \sum_{m=M(N)+1}^{2 N-1} \frac{1}{m^{2}} \leqslant \text { const } \cdot N \int_{M(N)}^{\infty} \frac{d x}{x^{2}}= \\
& =\text { const } \cdot \frac{N}{M(N)} \leqslant \text { const } \cdot \ln \ln N .
\end{aligned}
$$


Здесь в первом неравенстве мы воспользовались утверждением 3 леммы 3, второе неравенство следует из хорошо известного факта, что при $n \rightarrow \infty$

$$
\sum_{m=n}^{\infty} \frac{1}{m^{2}} \simeq \int_{n}^{\infty} \frac{d x}{x^{2}} \simeq \frac{1}{n}
$$

а в последнем неравенстве мы подставили определение (19) функции $M(N)$.

Объединяя полученные выкладки, получаем $\sup _{t \geqslant 0} I_{N, k, l}(t) \geqslant \sup _{t \geqslant 0} I_{N, k, l}^{1}(t)-\sup _{t \geqslant 0}\left|I_{N, k, l}^{2}(t)\right| \geqslant$ const $\cdot F_{N}(l)-$ const $\cdot \ln \ln N \geqslant$ const $\cdot F_{N}(l)$.

Оценка сверху. Теперь разобьем сумму в (18) по-другому,

$$
I_{N, k, l}(t)=J_{N, k, l}^{1}(t)+J_{N, k, l}^{2}(t):=\sum_{m \leqslant N / l}(\cdot)+\sum_{m>N / l}(\cdot),
$$

и оценим каждую из полученных частей по отдельности.

Имеем для первой суммы

$$
\sup _{t \geqslant 0}\left|J_{N, k, l}^{1}(t)\right| \leqslant \frac{\mathrm{const}}{N} \sum_{m \leqslant N / l} \frac{N l}{m}=\mathrm{const} \cdot l \cdot \sum_{m \leqslant N / l} \frac{1}{m} \leqslant \text { const } \cdot l \cdot \ln \frac{N}{l}=\mathrm{const} \cdot F_{N}(l) .
$$

Здесь в первом неравенстве мы воспользовались утверждениями 2 и 4 леммы 3, а в третьем - соотношением (21).

Для второй суммы имеем

$$
\sup _{t \geqslant 0}\left|J_{N, k, l}^{2}(t)\right| \leqslant \text { const } \cdot N \cdot \sum_{m=[N / l]}^{2 N-1} \frac{1}{m^{2}} \leqslant \text { const } \cdot N \int_{N / l}^{\infty} \frac{d x}{x^{2}}=\text { const } \cdot l \leqslant \text { const } \cdot F_{N}(l) .
$$

Здесь в первом неравенстве мы воспользовались утверждением 3 леммы 3, во втором - соотношением (22), а в последнем - определением $(6)$ функции $F_{N}(x)$. Объединяя полученные выкладки, выводим оценку сверху

$$
\sup _{t \geqslant 0}\left|I_{N, k, l}(t)\right| \leqslant \sup _{t \geqslant 0}\left|J_{N, k, l}^{1}(t)\right|+\sup _{t \geqslant 0}\left|J_{N, k, l}^{2}(t)\right| \leqslant \text { const } \cdot F_{N}(l) .
$$

3.3. Доказательство леммы 1. Имеем три уравнения для потенциала в точке $a=1 / N$ :

$$
\begin{aligned}
V(a) & =-c_{n} N^{n}+c_{m} N^{m} \\
V^{\prime}(a) & =0=n c_{n} N^{n+1}-m c_{m} N^{m+1} \\
V^{\prime \prime}(a) & =\kappa=-n(n+1) c_{n} N^{n+2}+m(m+1) c_{m} N^{m+2} .
\end{aligned}
$$

При этом в первом уравнении значение $V(a)$ неизвестно. Из уравнений $(23)$ и $(24)$ получаем, что

$$
c_{m}=\frac{n N^{-m} V(a)}{n-m}, \quad c_{n}=\frac{m N^{-n} V(a)}{n-m},
$$

а из уравнений $(24)$ и (25) имеем

$$
c_{m}=\frac{\kappa}{m(m-n)} N^{-2-m}, \quad c_{n}=\frac{\kappa}{n(m-n)} N^{-2-n} .
$$


Отсюда можно найти $V(a)$ :

$$
V(a)=-\frac{\kappa}{m n} \frac{1}{N^{2}}
$$

Нам нужна точка перегиба $b>a>0$, которую мы найдем из условия

$$
V^{\prime \prime}(b)=-n(n+1) c_{n} b^{-(n+2)}+m(m+1) c_{m} b^{-(m+2)}=0 .
$$

Имеем

$$
b=\frac{1}{N}\left(\frac{m+1}{n+1}\right)^{\frac{1}{m-n}}, \quad V^{\prime}(b)=n c_{n} b^{-n-1}-m c_{m} b^{-m-1} .
$$

Используя уравнение (24) вместе с уравнением (11) при $h=b$, мы найдем, что $\sigma(N)=f / \kappa$. Из формулы $(26)$ получаем

$$
\frac{f}{\kappa}=\frac{C}{N}, \quad C=C(n, m)=\frac{1}{m-n}\left(\left(\frac{m+1}{n+1}\right)^{-\frac{n+1}{m-n}}-\left(\frac{m+1}{n+1}\right)^{-\frac{m+1}{m-n}}\right) .
$$

Лемма доказана.

\section{Список литературы}

[1] O. Penrose, Markov Process. and Related Fields, 8:2 (2002), 351-364.

[2] M. Kardar, Problems and Solutions for Statistical Physics, MIT, Cambridge, 2008.

[3] V. A. Malyshev, Mosc. Math. J., 6:2 (2006), 353-358.

[4] G. Gallavotti (ed.), The Fermi-Pasta-Ulam Problem. A Status Report, Lecture Notes in Physics, 728, Springer, Berlin, 2008.

[5] О. Браун, Ю. Кившарь, Модель Френкеля-Конторовой. Концепции, методъ, приложения, Физматлит, М., 2008.

[6] A. Braides, M. Solci, E. Vitali, Netw. Heterog. Media, 2:3 (2007), 551-567.

[7] J. Braun, B. Schmidt, On the passage from atomistic systems to nonlinear elasticity theory, arXiv: 1107.4155 .

[8] E. Weinan, P. Ming, Arch. Ration. Mech. Anal., 183:2 (2007), 241-297.

[9] E. Weinan, P. Ming, Acta Math. Appl. Sin. Engl. Ser., 24:4 (2007), 529-550.

[10] М. Борн, Х. Кунь, Динамическая теория кристаллических решеток, ИЛ, М., 1958.

[11] А. Нивен, Числа рациональные и иррациональные, Мир, М., 1966.

[12] G. H. Hardy, E. M. Wright, An Introduction to the Theory of Numbers, Clarendon Press, Oxford, 1975. 